SCIENTIFIC REPORT

\title{
Topically administered timolol and dorzolamide reduce intraocular pressure and protect retinal ganglion cells in a rat experimental glaucoma model
}

\author{
M Seki, T Tanaka, H Matsuda, T Togano, K Hashimoto, J Ueda, T Fukuchi, H Abe
}

Br J Ophthalmol 2005;89:504-507. doi: 10.1136/bjo.2004.052860

\begin{abstract}
Aims: This study sought to elucidate the effects of timolol and dorzolamide on intraocular pressure (IOP) and retinal ganglion cell (RGC) death in an experimental model of glaucoma in rat.

Methods: Mild elevation of IOP was induced in rats by intracameral injection of India ink and subsequent laser trabecular photocoagulation. IOP was measured before the surgical procedures and weekly thereafter. Timolol $(0.5 \%)$, timolol XE $(0.5 \%)$, dorzolamide $(1 \%)$, and artificial tears (vehicle) were topically applied daily. Retinal sections were prepared for histology to determine RGC number.

Results: Timolol, timolol $X E$, and dorzolamide induced a significant reduction in IOP $(p<0.05)$ and counteracted the reduction in RGC number that occurred in vehicle treated glaucomatous eyes $(p<0.05)$. The coefficient of correlation between RGC number and IOP was significant in the dorzolamide treated group $(r=-0.908, p<0.005)$, but not in other groups $(p>0.05)$.

Conclusions: Both timolol formulation and dorzolamide reduced IOP and protected RGCs in a rat model of experimental glaucoma. It cannot be ruled out that timolol might protect RGCs by additional mechanisms other than simply lowering of IOP.
\end{abstract}

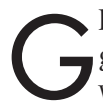
laucoma is a disorder featuring irreversible retinal ganglion cell (RGC) death which can be correlated with elevated intraocular pressure (IOP); however, the mechanism of RGC death in glaucoma is not fully understood. ${ }^{1}$ At present, the only well established treatment of glaucoma involves lowering the IOP. ${ }^{2}$ Nevertheless, randomised controlled clinical trials have demonstrated that even if medical and surgical treatments successfully lower the IOP, visual disturbances continue to progress in some patients. ${ }^{3-5}$ Thus, new methods of treating glaucoma by directly preventing RGC death are being investigated. Such neuroprotective strategies to protect RGCs by maintaining and enhancing their ability to resist stress and survive have been a focus of both physicians and researchers. However, most studies thus far have examined the neuroprotective potentials of various agents using RGCs in vitro or animal models of neural damage induced by optic nerve transsection, intraocular injection of glutamate, and transient ischaemic injury of the retina. In order to assess effectively the therapeutic value of such agents in glaucoma, however, they must be tested in animal models of glaucoma and then in randomised controlled clinical trials involving patients with glaucomatous optic neuropathy. ${ }^{6}$ Therefore, we investigated effects of timolol, a $\beta$ adrenoreceptor antagonist, and dorzolamide, a carbonic anhydrase inhibitor, upon IOP and
RGC number using an experimental glaucoma model to assess their neuroprotective effects.

\section{MATERIALS AND METHODS \\ Animal model of glaucoma}

All experimental procedures involving animals were performed in accordance with both the ARVO statement for the use of animals in ophthalmic and vision research and our institutional guidelines for care and use of laboratory animals. Adult male Wistar rats (Japan SLC, Hamamatsu, Japan), 9 weeks of age (250-300 g), were housed in standard lighting conditions ( 12 hour light and 12 hour dark cycle). Chronic elevation of IOP was induced in one eye of each animal by intracameral injection of India ink and subsequent laser trabecular photocoagulation 1 week after the injection. ${ }^{7}$ In each animal, the opposite eye was left unoperated. IOP was measured at the end of the light phase (17:00-18:00) with a Tono-Pen XL tonometer (BioRad, Santa Ana, CA, USA) with topical anaesthesia with a drop of $2 \%$ lidocaine applied to the eyes according to a previously described $\operatorname{method}^{8}$ once before the surgical induction of glaucoma and weekly thereafter. Rats with an IOP greater than $22 \mathrm{~mm} \mathrm{Hg}$ were classified as glaucomatous. The experimental schedule is summarised in figure $1 \mathrm{~A}$.

\section{Drug treatment}

Topical administration of antiglaucoma drugs was started 1 week after the laser photocoagulation and lasted for 3 weeks. Medications used in this study were timolol maleate (0.5\%, Timolol; Merck, Whitehouse Station, NJ, USA, $\mathrm{n}=6)$, timolol maleate gel forming solution $(0.5 \%$, Timolol XE; Merck, $\mathrm{n}=7$ ), and dorzolamide hydrochloride ( $1 \%$, Trusopt; Merck, $\mathrm{n}=7$ ). Ten $\mu \mathrm{l}$ of eye drop was applied twice, once, and three times daily, respectively for each drug, following clinical protocols in Japan. Artificial tears (Mytear; Senju Pharmaceutical, Osaka, Japan, $\mathrm{n}=8$ ) were used as vehicle (three times daily), and unoperated eyes from the vehicle treated animals served as controls $(n=7)$.

\section{Histopathology}

Animals were deeply anaesthetised by chloral hydrate and sacrificed by decapitation. Posterior eye cups were fixed with $4 \%$ paraformaldehyde, dehydrated, and embedded in paraffin. Radial sections $(4 \mu \mathrm{m})$ were stained with Mayer's haematoxylin solution (Wako, Osaka, Japan) and Eosin Alcohol Solution (Wako). Specimens were observed at a distance of $1.5 \mathrm{~mm}$ from the centre of the optic nerve head under an AxioPhoto2 microscope (Carl Zeiss, Jena, Germany), and photomicrographs were captured using a digital camera (AxioCam; Carl Zeiss). Cells in the ganglion

Abbreviations: BDNF, brain derived neurotrophic factor; IOP, intraocular pressure; RGC, retinal ganglion cell 

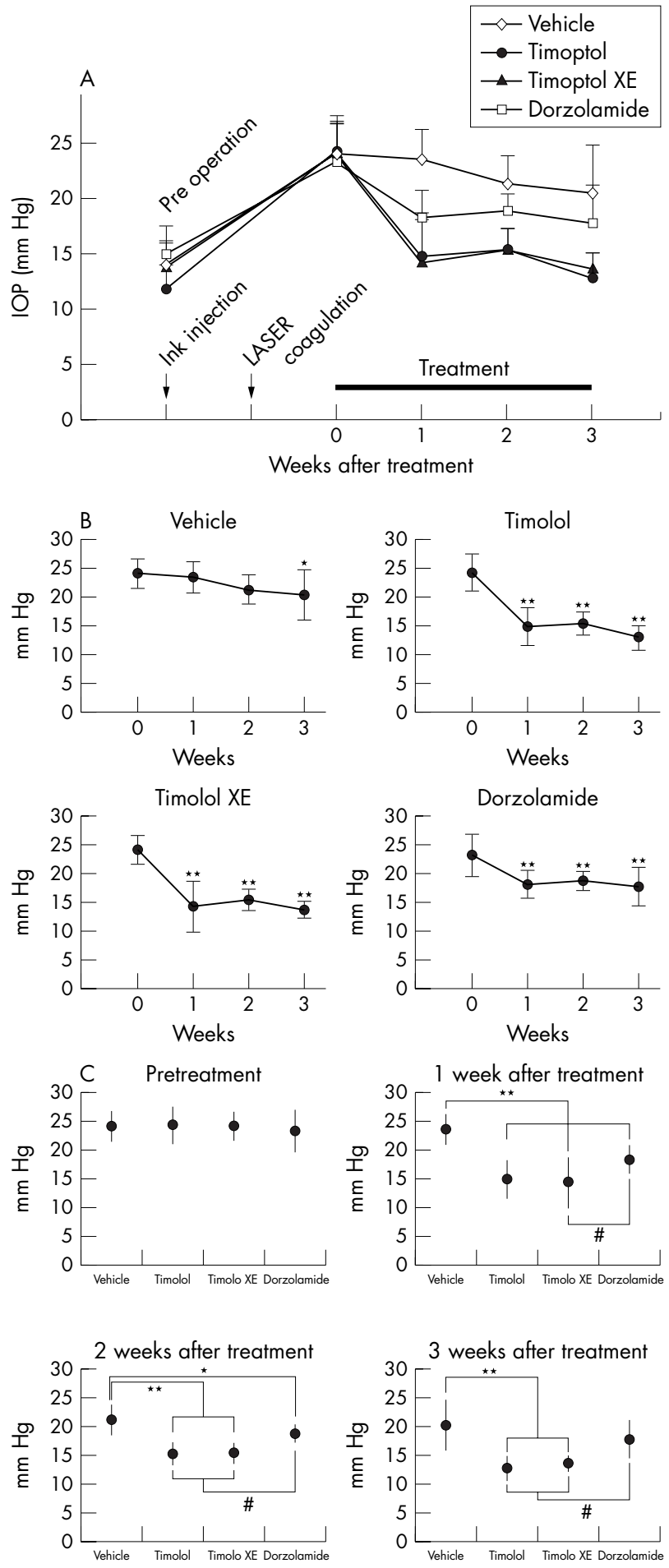

Figure 1 (A) The experimental schedule is summarised schematically. Chronic elevation of IOP was induced in rats by intracameral injection of India ink and subsequent laser trabecular photocoagulation. IOP was measured with a Tono-Pen XL tonometer. Topical administration of antiglaucoma drugs was started 1 week after the laser photocoagulation and lasted for 3 weeks thereafter. (B) Time course of mean IOP level in each experimental group is shown. Statistical significance was analysed by paired $t$ test. ${ }^{*} p<0.05,{ }^{* *} p<0.005$ compared with pretreatment IOP values. (C) Mean IOP levels were compared between drugs at each time point. One way ANOVA followed by Fisher's PLSD multiple comparison test gave ${ }^{*} p<0.05,{ }^{* *} p<0.005$ in comparison with vehicle treated eyes, and $\# p<0.05$ with dorzolamide treated eyes. cell layer were counted and reported as a density of RGCs (cells/mm). ${ }^{9}$ Histopathological examinations were repeated at least twice.

\section{Statistical analysis}

All values are expressed as means (SD). Statistical significance was analysed either by paired $t$ test or one way ANOVA followed by Fisher's PLSD multiple comparison test, as indicated. Pearson's correlation coefficient analysis was used to investigate the correlation between IOP and RGC number. Values of $\mathrm{p}<0.05$ were taken to be significant.

\section{RESULTS}

Induction of ocular hypertension in an animal model of glaucoma

Preoperative IOP was not significantly different between experimental groups (control, 15.4 (SD 3.9) mm Hg; vehicle, 15.8 (3.7) $\mathrm{mm} \mathrm{Hg}$; timolol, 14.6 (3.9) $\mathrm{mm} \mathrm{Hg}$; timolol XE, 15.4 (4.9) mm Hg; dorzolamide, 15.8 (3.7) mm Hg; p = 0.88, one way ANOVA). One week after trabecular photocoagulation, IOP was significantly elevated in glaucomatous eyes (vehicle, 24.1 (2.6) $\mathrm{mm} \mathrm{Hg}$; timolol, 24.3 (3.2) $\mathrm{mm} \mathrm{Hg}$; timolol XE, 24.2 (2.5) mm Hg; dorzolamide, 23.3 (3.6) $\mathrm{mm} \mathrm{Hg}$ ) compared to control, unoperated eyes (15.3 (3.1) $\mathrm{mm} \mathrm{Hg}, \mathrm{p}<0.05$, one way ANOVA followed by Fisher's PLSD multiple comparison test).

\section{The effect of topically applied antiglaucoma drugs on} IOP in the rat glaucoma model

Time course of mean IOP level in each group is shown in figure $1 \mathrm{~B}$. Treatment with timolol, timolol $\mathrm{XE}$, and dorzolamide all resulted in a decrease in IOP $(\mathrm{p}<0.01$, paired $t$ test $)$. However, IOP was also reduced after 3 weeks even in the vehicle treated group $(\mathrm{p}<0.05)$.

Before drug treatment, the IOP of glaucomatous eyes was not significantly different between experimental groups $(\mathrm{p}=0.92$, one way ANOVA) (fig $\mathrm{lC}$ ). Both timolol and timolol XE significantly lowered IOP $(\mathrm{p}<0.001$ in comparison with the vehicle treated group, one way ANOVA followed by Fisher's PLSD multiple comparison test). Dorzolamide also reduced IOP after 1 and 2 weeks of treatment $(p<0.05)$. The effect on IOP level of timolol and timolol XE was significantly greater than that of dorzolamide, indicating that they are more effective in lowering IOP in this animal model $(\mathrm{p}<0.05$ in comparison with the dorzolamide treated group, one way ANOVA followed by Fisher's PLSD multiple comparison test).

\section{The therapeutic effect of antiglaucoma drugs on RGCs} in the rat glaucoma model

In order to analyse the therapeutic efficacy of antiglaucoma drugs on RGC degeneration in this animal model, radial sections of the retina were prepared (fig 2), and RGC number was counted (table 1). In glaucomatous eyes, RGC number was markedly decreased (control $v$ vehicle, $\mathrm{p}<0.005$, one way ANOVA followed by Fisher's PLSD multiple comparison test). Treatment with antiglaucoma drugs efficiently prevented to varying extents this decrease in RGC number $(\mathrm{p}<0.05$ in comparison with the vehicle treated eyes). Although timolol and dorzolamide treated groups did show reduced RGC number when compared to control eyes $(p<0.05)$, no statistically significant difference was observed between control and timolol XE treated eyes $(\mathrm{p}=0.09)$.

\section{Correlation between IOP and RGC number in the rat glaucoma model}

A weak negative correlation was present between IOP and RGC count when Pearson's correlation coefficient analysis was made with all glaucomatous eyes $(r=-0.377, \mathrm{p}<0.05)$ (fig 3). Although a highly significant negative relation was 

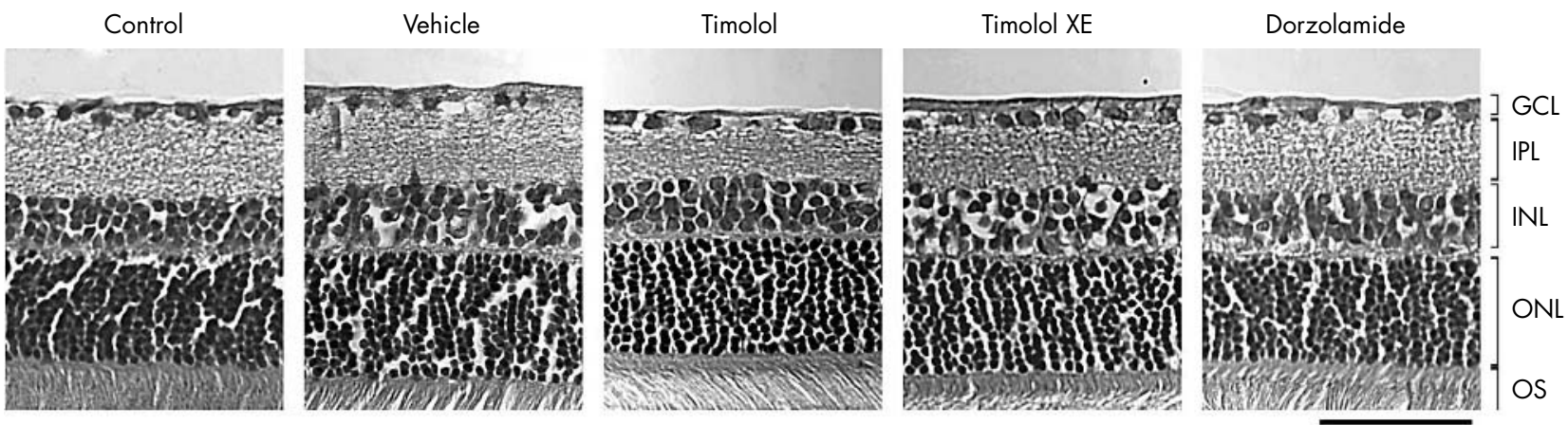

Figure 2 To determine RGC number in retinas of experimental animals, cells in the ganglion cell layer were counted in radial sections. GCL, ganglion cell layer; IPL, inner plexiform layer; INL, inner nuclear layer; OPL, outer plexiform layer; ONL, outer nuclear layer. Scale bar $=50 \mu \mathrm{m}$.

found between IOP and RGC number in the dorzolamide treated group $(r=-0.908, \mathrm{p}<0.005)$, no such statistically significant correlation was found in either of the other two treatment groups $(\mathrm{p}>0.05)$.

\section{DISCUSSION}

In the present study, we assessed the effects of timolol and dorzolamide upon IOP and RGCs in a rat model of experimental glaucoma, ${ }^{7}$ whose reproducibility was demonstrated by others. ${ }^{10}$ Our present study showed that both timolol and dorzolamide reduced IOP (fig 1) and protected RGCs (fig 2 and table 1). Further correlation analysis revealed that IOP was correlated with RGC damage in dorzolamide treated, but not in timolol and timolol XE treated eyes. Thus, dorzolamide might protect RGCs from degeneration mainly in an IOP dependent manner under the conditions used in this study. In contrast with our study, however, dorzolamide was not effective in preventing RGC death in DBA/2J mice that had spontaneously developed elevated IOP. ${ }^{11}$ Although lower IOP preserves visual field of patients with primary open angle glaucoma, ${ }^{12}$ some studies failed to demonstrate correlation between IOP and visual field sensitivity in timolol treated glaucomatous individuals..$^{13}{ }^{14}$ Similarly, we did not see any sign of such correlation in the animal model of glaucoma in the present study, leaving open the mechanism by which timolol protected RGCs. There have been some previous reports of the potential neuroprotective effects of $\beta$ adrenergic antagonists as assessed both in vitro ${ }^{9}$ and in an in vivo animal model of retinal insult induced by transient ischaemia. ${ }^{9}{ }^{15}$ Thus, it cannot be ruled out that timolol might protect RGCs by additional mechanisms other than lowering IOP in the present study.

One possibility for how topically applied timolol rescues RGCs involves brain derived neurotrophic factor (BDNF), which is a strong neuroprotectant of RGCs. ${ }^{16}{ }^{17}$ Topically

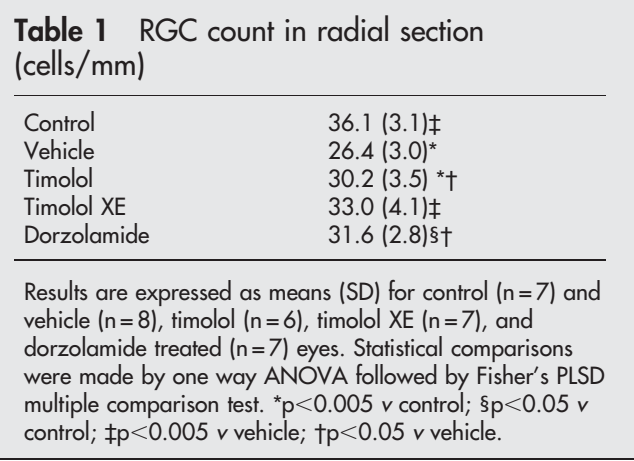

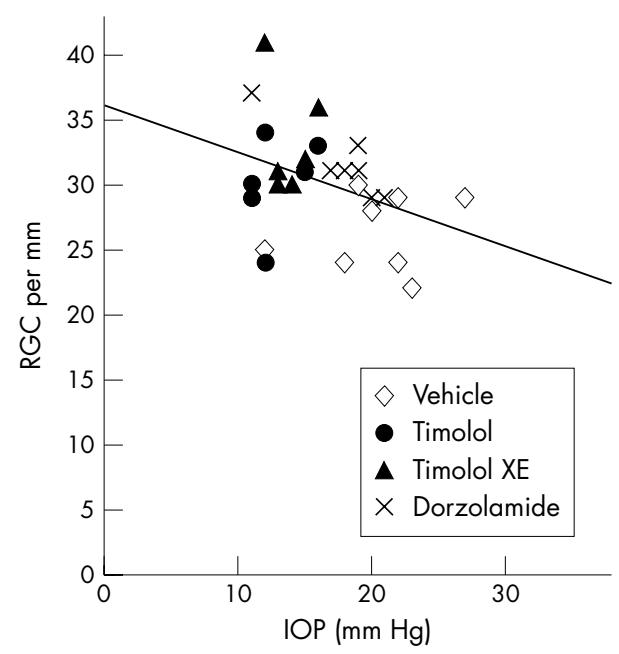

Figure 3 Pearson's correlation coefficient analysis was used to investigate the correlation between IOP and RGC number. IOP and RGC number were weakly negatively correlated for all glaucomatous eyes $(r=-0.377, \mathrm{p}<0.05)$.

applied betaxolol, a $\beta$ adrenergic antagonist, was shown to stimulate BDNF mRNA expression in the retina. ${ }^{18}$ Therefore, upregulation of BDNF expression upon topical administration of timolol might protect RGCs. BDNF is one of the most attractive candidate neuroprotective drugs for glaucoma; its therapeutic potential has been demonstrated in a rat glaucoma model. ${ }^{19}$ In that study, BDNF was injected directly into the eyeball, but frequent injections into patients' vitreous space are not practical. We previously demonstrated that BDNF is expressed endogenously in the rat retina. ${ }^{20}$ The discovery raises a more practical therapeutic approach: induction of endogenous BDNF by external administration of agents with a smaller molecular weight, such as $\beta$ adrenergic antagonists, may be useful to treat glaucoma.

\section{ACKNOWLEDGEMENTS}

This work was supported by a research grant from the Ministry of Health, Labor and Welfare, and in part by unrestricted research grants from Banyu Pharmaceutical Co, Ltd.

\section{Authors' affiliations}

M Seki, T Tanaka, H Matsuda, T Togano, K Hashimoto, J Ueda, T Fukuchi, H Abe, Division of Ophthalmology and Visual Science, Graduated School of Medical and Dental Sciences, Niigata University, Niigata, Japan 
Correspondence to: Takeo Fukuchi, MD, PhD, Division of Ophthalmology and Visual Science, Graduated School of Medical and Dental Sciences, Niigata University, 1-757 Asahimachi, Niigata,

Niigata 951-8585, Japan; ffuku@med.niigata-u.ac.jp

Accepted for publication 3 September 2004

\section{REFERENCES}

1 Quigley HA. Neuronal death in glaucoma. Prog Retin Eye Res 1999; 18:39-57.

2 Goldberg I. Relationship between intraocular pressure and preservation of visual field in glaucoma. Surv Ophthalmol 2003;48(Suppl 1):S3-7.

3 Collaborative Normal-Tension Glaucoma Study Group. Comparison of glaucomatous progression between untreated patients with normal-tension glaucoma and patients with therapeutically reduced intraocular pressures. Am J Ophthalmol 1998;126:487-97.

4 Collaborative Normal-Tension Glaucoma Study Group. The effectiveness of intraocular pressure reduction in the treatment of normal-tension glaucoma. Am J Ophthalmol 1998;126:498-505.

5 The AGIS Investigators. The Advanced Glaucoma Intervention Study (AGIS): 7. The relationship between control of intraocular pressure and visual field deterioration. Am J Ophthalmol 2000;130:429-40.

6 Levin LA. Retinal ganglion cells and neuroprotection for glaucoma. Surv Ophthalmol 2003;48(Suppl 1):S21-4.

7 Ueda J, Sawaguchi S, Hanyu T, et al. Experimental glaucoma model in the rat induced by laser trabecular photocoagulation after an intracameral injection of India ink. Jpn J Ophthalmol 1998:42:337-44.

8 Moore CG, Milne ST, Morrison JC. Noninvasive measurement of rat intraocular pressure with the Tono-Pen. Invest Ophthalmol Vis Sci 1993;34:363-69.

9 Goto W, Ota T, Morikawa N, et al. Protective effects of timolol against the neuronal damage induced by glutamate and ischemia in the rat retina. Brain Res 2002;958:10-19.
10 Ishii Y, Kwong JM, Caprioli J. Retinal ganglion cell protection with geranylgeranylacetone, a heat shock protein inducer, in a rat glaucoma model. Invest Ophthalmol Vis Sci 2003;44:1982-92.

11 Schuettauf F, Quinto K, Naskar R, et al. Effects of anti-glaucoma medications on ganglion cell survival: the DBA/2J mouse model. Vis Res 2002;42:2333-37.

12 Mao LK, Stewart WC, Shields MB. Correlation between intraocular pressure control and progressive glaucomatous damage in primary open-angle glaucoma. Am J Ophthalmol 1991;111:51-5.

13 Messmer C, Flammer J, Stumpfig D. Influence of betaxolol and timolol on the visual fields of patients with glaucoma. Am J Ophthalmol $1991 ; 112: 678-81$.

14 Collignon-Brach J. Longterm effect of topical beta-blockers on intraocular pressure and visual field sensitivity in ocular hypertension and chronic openangle glaucoma. Surv Ophthalmol 1994;38(Suppl):S149-55.

15 Wood JP, Schmidt KG, Melena J, et al. The beta-adrenoceptor antagonists metipranolol and timolol are retinal neuroprotectants: comparison with betaxolol. Exp Eye Res 2003;76:505-16.

16 Johnson JE, Barde YA, Schwab M, et al. Brain-derived neurotrophic factor supports the survival of cultured rat retinal ganglion cells. J Neurosci 1986;6:3031-38

17 Mansour-Robaey S, Clarke DB, Wang YC, et al. Effects of ocular injury and administration of brain-derived neurotrophic factor on survival and regrowth of axotomized retinal ganglion cells. Proc Natl Acad Sci USA 1994;91:1632-6.

18 Wood JP, DeSantis L, Chao HM, et al. Topically applied betaxolol attenuates ischaemia-induced effects to the rat retina and stimulates BDNF mRNA. Exp Eye Res $2001 ; 72: 79-86$.

19 Ko ML, Hu DN, Ritch R, et al. The combined effect of brain-derived neurotrophic factor and a free radical scavenger in experimental glaucoma. Invest Ophthalmol Vis Sci 2000;41:2967-71.

20 Seki M, Nawa H, Fukuchi T, et al. BDNF is upregulated by postnatal development and visual experience: quantitative and immunohistochemical analyses of BDNF in the rat retina. Invest Ophthalmol Vis Sci 2003;44:3211-18. 